\title{
Nomograms for Prediction of Outcome With or Without Adjuvant Radiation Therapy for Patients With Endometrial Cancer: A Pooled Analysis of PORTEC-1 and PORTEC-2 Trials
}

Citation for published version (APA):

Creutzberg, C. L., van Stiphout, R. G. P. M., Nout, R. A., Lutgens, L. C. H. W., Juergenliemk-Schulz, I. M., Jobsen, J. J., Smit, V. T. H. B. M., \& Lambin, P. (2015). Nomograms for Prediction of Outcome With or Without Adjuvant Radiation Therapy for Patients With Endometrial Cancer: A Pooled Analysis of PORTEC-1 and PORTEC-2 Trials. International Journal of Radiation Oncology Biology Physics, 91(3), 530-539. https://doi.org/10.1016/j.jijobp.2014.11.022

Document status and date:

Published: 01/03/2015

DOI:

10.1016/j.jijrobp.2014.11.022

Document Version:

Publisher's PDF, also known as Version of record

Document license:

Taverne

Please check the document version of this publication:

- A submitted manuscript is the version of the article upon submission and before peer-review. There can be important differences between the submitted version and the official published version of record. People interested in the research are advised to contact the author for the final version of the publication, or visit the DOI to the publisher's website.

- The final author version and the galley proof are versions of the publication after peer review.

- The final published version features the final layout of the paper including the volume, issue and page numbers.

Link to publication

\footnotetext{
General rights rights.

- You may freely distribute the URL identifying the publication in the public portal. please follow below link for the End User Agreement:

www.umlib.nl/taverne-license

Take down policy

If you believe that this document breaches copyright please contact us at:

repository@maastrichtuniversity.nl

providing details and we will investigate your claim.
}

Copyright and moral rights for the publications made accessible in the public portal are retained by the authors and/or other copyright owners and it is a condition of accessing publications that users recognise and abide by the legal requirements associated with these

- Users may download and print one copy of any publication from the public portal for the purpose of private study or research.

- You may not further distribute the material or use it for any profit-making activity or commercial gain

If the publication is distributed under the terms of Article 25fa of the Dutch Copyright Act, indicated by the "Taverne" license above, 


\title{
Nomograms for Prediction of Outcome With or Without Adjuvant Radiation Therapy for Patients With Endometrial Cancer: A Pooled Analysis of PORTEC-1 and PORTEC-2 Trials
}

\author{
Carien L. Creutzberg, MD, PhD, ${ }^{*}$ Ruud G.P.M. van Stiphout, MSc, PhD, ${ }^{\dagger}$ \\ Remi A. Nout, MD, PhD, ${ }^{*}$ Ludy C.H.W. Lutgens, MD, PhD, \\ Ina M. Jürgenliemk-Schulz, MD, PhD, Jan J. Jobsen, MD, PhD, \\ Vincent T.H.B.M. Smit, MD, PhD, and Philippe Lambin, MD, PhD ${ }^{\dagger}$ \\ *Department of Clinical Oncology, Leiden University Medical Center, Leiden, The Netherlands; \\ ${ }^{\dagger}$ Department of Radiation Oncology, MAASTRO, GROW, University Medical Centre Maastricht, \\ Maastricht, The Netherlands; ${ }^{\ddagger}$ Department of Radiation Oncology, University Medical Center Utrecht, \\ Utrecht, The Netherlands; ${ }^{\circledR}$ Department of Radiotherapy, Medisch Spectrum Twente, Enschede, The \\ Netherlands; and "Department of Pathology, Leiden University Medical Center, Leiden, The \\ Netherlands
}

Received Jul 8, 2014, and in revised form Nov 8, 2014. Accepted for publication Nov 12, 2014.

\author{
Summary \\ To facilitate treatment deci- \\ sion support for individual \\ patients with early stage \\ endometrial cancer, nomo- \\ grams were developed from \\ the PORTEC-1 and -2 data- \\ bases $(\mathrm{N}=1240$ patients) to \\ predict the individual pa- \\ tient's risk of locoregional \\ relapse, distant relapse, \\ overall survival, and disease- \\ free survival. These
}

Background: Postoperative radiation therapy for stage I endometrial cancer improves locoregional control but is without survival benefit. To facilitate treatment decision support for individual patients, accurate statistical models to predict locoregional relapse (LRR), distant relapse (DR), overall survival (OS), and disease-free survival (DFS) are required.

Methods and Materials: Clinical trial data from the randomized Post Operative Radiation Therapy for Endometrial Cancer (PORTEC-1; $\mathrm{N}=714$ patients) and PORTEC-2 ( $\mathrm{N}=427$ patients) trials and registered group (grade 3 and deep invasion, $\mathrm{n}=99)$ were pooled for analysis $(\mathrm{N}=1240)$. For most patients $(86 \%)$ pathology review data were available; otherwise original pathology data were used. Trial variables which were clinically relevant and eligible according to data constraints were age, stage, given treatment (pelvic external beam radiation therapy (EBRT), vaginal brachytherapy (VBT), or no adjuvant treatment, FIGO histological grade, depth of invasion, and lymph-vascular invasion (LVSI). Multivariate analyses were based on
Reprint requests to: Carien L. Creutzberg, MD, PhD, Department of Clinical Oncology, K1-P, Leiden University Medical Center, Albinusdreef 2, PO Box 9600, 2300 RC Leiden, The Netherlands. Tel: 3171526 5120; E-mail: c.l.creutzberg@lumc.nl

This work was presented in part at the European Cancer Congress ECCO17 ESMO38, -ESTRO32, Amsterdam, The Netherlands, September 2013.
Conflict of interest: none.

The PORTEC studies were supported by Dutch Cancer Society grants CKVO 90-01 and 2001-04.

Supplementary material for this article can be found at www.redjournal.org.

Acknowledgment-We thank the radiation oncologists, gynecologists, pathologists, and data managers at the participating centers. 
nomograms are indispensable for patient counseling and shared decision making, especially as they include the effect of external beam radiation therapy or vaginal brachytherapy on the risks of locoregional relapse and disease-free survival.
Cox proportional hazards regression model. Predictors were selected based on a backward elimination scheme. Model results were expressed by the c-index (0.5-1.0; random to perfect prediction). Two validation sets $(n=244$ and 291 patients) were used.

Results: Accuracy of the developed models was good, with training accuracies between 0.71 and 0.78. The nomograms validated well for DR (0.73), DFS (0.69), and OS (0.70), but validation was only fair for LRR (0.59). Ranking of variables as to their predictive power showed that age, tumor grade, and LVSI were highly predictive for all outcomes, and given treatment for LRR and DFS. The nomograms were able to significantly distinguish low- from high-probability patients for these outcomes.

Conclusions: The nomograms are internally validated and able to accurately predict long-term outcome for endometrial cancer patients with observation, pelvic EBRT, or VBT after surgery. These models facilitate decision support in daily clinical practice and can be used for patient counseling and shared decision making, selecting patients who benefit most from adjuvant treatment, and generating new hypotheses. (C) 2015 Elsevier Inc.

\section{Introduction}

Endometrial cancer (EC) is the most common gynecological cancer in developed countries. Surgery is the cornerstone of treatment. Postoperative pelvic external beam radiation therapy (EBRT) for early stage EC has been shown in multiple randomized trials to significantly increase locoregional control but has no impact on overall survival (OS) (1,2). The indication for adjuvant RT is tailored to the risk of recurrence. This spares most EC patients with a favorable prognosis the negative effects of pelvic EBRT, and significantly reduces the risk of disease recurrence, with its associated anxiety and stress, and the toxicities of salvage treatment in the minority of patients who are at higher risk (3). Using pelvic EBRT for patients with low-risk disease may even negatively affect their eventual outcome $(2,4)$.

For EC patients with high-intermediate risk factors, vaginal brachytherapy (VBT) alone has been shown to result in excellent vaginal cancer control, without the shortand long-term toxicities of $\operatorname{EBRT}(5,6)$. To refine the indication for adjuvant therapy among patients with risk factors and to identify the occasional patient with higher risk among those with a low-risk profile, studies of molecular genetic risk factors are increasingly being done, and some have shown promising results $(7,8)$.

When patients are asked about their treatment preferences, in most cases they favor adjuvant treatment to ensure they have used all possible treatments to reduce the risk of cancer recurrence. In addition, they do wish to be involved in clinical decision making and be informed about the risk and benefits of alternative treatment options, and make an informed, joint decision together with their physician $(9,10)$.

Recently, treatment decision support nomograms have been developed by several groups to facilitate patient information and counseling based on their individual situation and individual risks of local, regional, and distant recurrence. Such systems have been based on large databases of historical patients and have incorporated various patient and tumor characteristics and outcomes (11-13). The Australian nomogram, which is also available as a mobile phone application, has been based on results of 2097 patients and is focused on assessing the individual patient's risk of isolated locoregional and distant recurrence (12). The Memorial Sloan-Kettering Cancer Center nomogram informs on the risk of 3-year OS, without differentiating between local and distant relapse (DR) (11). The most recent nomogram, developed by AlHilli et al (13), is focused on assessing the individual patient's risk of (microscopic) pelvic lymph node metastases, to be used for patient counseling regarding the indication for second surgery; they used clinical data from 883 patients treated over a 10-year time period.

None of the previous studies has been able to include adjuvant treatment modalities in the risk prediction models, as adjuvant treatment had not been used consistently over the long study periods. This would require a patient cohort in which adjuvant treatment was used consistently and preferably randomly allocated. For most patients with early stage disease, the inclusion of outcomes with or without adjuvant RT, specifically pelvic EBRT and VBT, would be highly desirable to facilitate individual patient counseling on the risks and benefits of using adjuvant treatment in their particular situation.

We combined the databases from the randomized Post Operative Radiation Therapy for Endometrial Cancer (PORTEC)-1 and PORTEC-2 trials and used this pooled patient data set, in which adjuvant treatment had been randomly allocated to develop such treatment decision support nomograms, including outcomes with and without adjuvant treatment. 


\section{Methods and Materials}

\section{Patient and tumor characteristics}

Clinical trial data from the PORTEC-1 $(\mathrm{N}=714$ patients) and PORTEC- $2(\mathrm{~N}=427$ patients) trials and the PORTEC registration group with grade 3 and deep invasion $(\mathrm{N}=99$ patients) were pooled for this analysis, providing a total number of 1240 patients. In the PORTEC-1 trial, patients with stage I EC with low-intermediate and highintermediate risk features were randomly allocated to adjuvant EBRT and observation after surgery $(14,15)$ In the PORTEC-2 trial, patients with stage I EC (and International Federation of Gynecology and Obstetrics [FIGO] 1988 stage IIA, which are classified as stage I in the 2009 FIGO staging) with high-intermediate risk factors were randomly allocated to pelvic EBRT or VBT (5). During the inclusion period of the PORTEC-1 trial, stage I patients with highrisk factors (grade 3 with deep myometrial invasion) were not randomized but were registered, and all received adjuvant pelvic EBRT (16). Finally, 2 validation sets were used. The first was obtained from the Maastricht radiation oncology retrospective database, including all patients who fitted the inclusion criteria for PORTEC-1 and PORTEC-2 trials and the high-risk registry group (but who were not included in those trials) and who had been treated between 1978 and $2010(n=244$ patients). The second validation set was obtained from the Medisch Spectrum Twente Enschede endometrial carcinoma database, which included all patients who fitted the same criteria and had been treated for FIGO 1988 stages I to IIA between August 1988 and March 2014 , with exclusion of those patients who had participated in one of the PORTEC trials $(n=291$ patients).

Patient and tumor characteristics and outcome data were obtained from the trial databases (Tables 1 and 2) $(5,15)$. Data from the pathology review from both trials were used if available (80\% for PORTEC-1 and $90 \%$ for PORTEC-2; overall $86 \%$ ); otherwise, original pathology data were used. Age was calculated at the time of randomization. Time-toevent analyses were done from the date of surgery, as this date was available for all patients, including the registered and validation groups. Patients lost to follow-up $(n=42)$ were considered censored at the day of last follow-up. Median follow-up for patients alive was 160 months for PORTEC-1 and 89 months for PORTEC-2 and 213 and 90 months for the Maastricht and Enschede validation sets, respectively.

Initially, the following variables were selected as clinically relevant for follow-up outcome: FIGO 1988 (sub) stage, age, FIGO grade, depth of myometrial invasion, width of the uninvolved myometrium, distance between the point of deepest tumor invasion to the serosal surface, lymph-vascular space invasion and/or angioinvasion, involvement of the cornuae (uterine cornua or horns), and treatment given. Variables that were not used because the percentage of missing values was too high or variation
Table 1 Trial and treatment information

\begin{tabular}{lc}
\hline \multicolumn{1}{c}{ Parameter } & No. of patients $(\%)$ \\
\hline Treatment group & \\
PORTEC-I & $714(57.6)$ \\
PORTEC-II & $427(34.4)$ \\
Registered group* & $99(8.0)$ \\
Given treatment & \\
EBRT & $648(52.3)$ \\
VBT & $214(17.3)$ \\
Observation & $378(30.4)$ \\
Randomized treatment & \\
EBRT & $667(53.8)$ \\
VBT & $213(17.2)$ \\
Observation & $360(29.0)$ \\
Validation group Maastricht & $150(61.5)$ \\
EBRT & $94(38.5)$ \\
VBT & $0(0.0)$ \\
Observation & \\
Validation group Enschede & $203(69.8)$ \\
EBRT & $88(30.2)$ \\
VBT & $0(0.0)$ \\
Observation & \\
\hline Abbreviations: EBRT = pelvic external beam radiation therapy; \\
VBT = vaginal brachytherapy. \\
$*$ Registered patients with grade 3 and outer myometrial invasion \\
were all given EBRT.
\end{tabular}

power of values was too low included FIGO 1988 (sub) stage (deterministic with myometrial invasion and grade), width of the uninvolved myometrium, and distance from the serosa (too many missing values). Peritoneal cytology was not taken into account because of the rarity of malignant findings.

Clinical, pathologic, and treatment variables were tested for significance. The variables that were used in the final analysis were age (as continuous variable), treatment given (pelvic EBRT, VBT, or no adjuvant treatment), FIGO histological grade, depth of myometrial invasion, involvement of the cornuae uteri (where the myometrium is thinnest), and lymph-vascular space invasion (LVSI) and/or angioinvasion. Outcome data were defined as vaginal relapse (VR), pelvic relapse (PR), combined locoregional relapse (LRR [vaginal and/or pelvic]), DR, OS, and disease-free survival (DFS).

OS was taken from date of randomization or surgery (for nontrial patients) with failure defined as death regardless of the cause and censored at the date of last contact for patients still alive; for DFS, with failure taken as death or relapse, whichever occurred first, and censored at the date of last contact for patients still alive and disease free.

\section{Statistical methods}

Any missing values were imputed by the expectationmaximization algorithm. Data were normalized (mean, 0; standard deviation, 1) before any analysis was performed. 
Table 2 Patient characteristics of the initial and final pooled trial cohort and the validation sets

\begin{tabular}{|c|c|c|c|c|}
\hline & $\underline{\text { Initial pooled trial cohort }}{ }^{*}$ & $\underline{\text { Final trial cohort }}{ }^{*}$ & $\underline{\text { Validation Maastro }}$ & Validation Enschede \\
\hline Characteristic & $\mathrm{N}(\%)$ & $\mathrm{n}(\%)$ & $\mathrm{n}(\%)$ & $\mathrm{n}(\%)$ \\
\hline Total & 1240 & 1240 & 244 & 291 \\
\hline Median & 68.0 & 68.0 & 65.8 & 68.0 \\
\hline Range & $35.2-92.2$ & $35.2-92.2$ & $19.5-86.8$ & $44.0-92.0$ \\
\hline \multicolumn{5}{|c|}{ FIGO histological grade } \\
\hline 3 & $207(16.7)$ & $254(20.5)$ & $20(8.2)$ & $91(31.3)$ \\
\hline Unknown & $0(0)$ & $0(0)$ & $7(2.9)$ & $6(2.1)$ \\
\hline \multicolumn{5}{|c|}{ Myometrial invasion } \\
\hline$<50 \%$ & $352(28.4)$ & $368(29.7)$ & $110(45.1)$ & $77(26.5)$ \\
\hline$>50 \%$ & $888(71.6)$ & $872(70.3)$ & $131(53.7)$ & $214(73.5)$ \\
\hline Unknown & $42(3.4)$ & $30(2.4)$ & $27(11.1)$ & $0(0)$ \\
\hline \multicolumn{5}{|c|}{ Vascular invasion } \\
\hline No & $1046(84.4)$ & $1106(89.2)$ & $192(78.7)$ & $246(84.5)$ \\
\hline Yes & $111(9.0)$ & $117(9.4)$ & $52(21.3)$ & $45(15.5)$ \\
\hline Unknown & $83(6.7)$ & $17(1.4)$ & $0(0)$ & $0(0)$ \\
\hline \multicolumn{5}{|c|}{ Radiation therapy } \\
\hline None & $378(30.5)$ & $378(30.5)$ & $0(0)$ & $0(0)$ \\
\hline EBRT & $648(52.3)$ & $648(52.3)$ & $150(61.5)$ & $203(69.8)$ \\
\hline VBT & $214(17.3)$ & $214(17.3)$ & $94(38.5)$ & $88(30.2)$ \\
\hline
\end{tabular}

Multivariate analyses were based on the Cox proportional hazard model, and model performances using the censored outcome data were assessed by the c-index. This index has properties similar to area under the receiver operating characteristic curve $(0.5$, random prediction; 1 , perfect prediction). The variable "given treatment," was replaced by 2 dummy variables for EBRT and VBT because the specific order of the values does not have a meaning (ie, the variable is not ordinal). Age was treated as a continuous variable and FIGO grade as an ordinal variable, and the other invasion-related variables were converted to binary variables (no, yes), which meant they were nominal in the analysis.

Identifying the predictors, that is, the variables that have a significant association with outcome, was done in a multivariate setting, using a backward elimination process. In this method, one starts with all variables in the multivariate model and tests for significance. In each subsequent iteration, the least significant variable was excluded, and the new combination of variables was tested again. This process was repeated until all variables were predictive $(\alpha=.05)$. This final predictor set was used to develop the nomograms, which are visual representations of the trained Cox models. Performance of the nomograms was calculated by using bootstrapping in which the evaluated data set was tested 1000 times, and each time, data were randomly resampled (patients could occur in the data set more than once), and c-index was calculated. The final step was to compute the average bootstrapped performance and its $95 \%$ confidence interval. Calibration, that is, predicted event rates versus observed event rates, was assessed by plotting these quantities in subgroups with equal numbers of patients (training: 9; subgroups, merged validation: 6 subgroups). The Hosmer-Lemeshow statistic was used to test significance of the calibration (in this test, significant $P$ values relate to poor calibration).

\section{Results}

\section{Patient outcomes}

Among the total of 1240 patients in our analysis, 648 (52.3\%) had received adjuvant pelvic EBRT, 214 (17.3\%) had received VBT, and 378 (30.4\%) had been observed after surgery. Further treatment information and trial origins are reported in Table 1. Patient characteristics of the pooled trial cohorts and validation sets are shown in 
Table 2. Rates of vaginal and pelvic recurrence, distant metastases, and OS and DFS are shown in Table 3.

\section{Nomogram development}

For the development of the nomograms, the complete pooled dataset with the PORTEC patients and the PORTEC registration group with grade 3 and deep myometrial invasion was used $(\mathrm{N}=1240$ patients). These data were updated for the pathology review (Table 2). The backwards elimination method for predictor selection resulted in multiple predictors for the 4 assessed outcomes: LRR, DR, DFS, and OS (Table 4). The predictors age, FIGO grade, and LVSI were consistently selected for all outcomes. Age (in years) had the highest hazard ratios (HR) for DFS (HR $=1.77)$ and $\mathrm{OS}(\mathrm{HR}=1.94)$ compared to the other predictors, and FIGO grade had the highest HR for LRR (HR $=1.64)$ and $\mathrm{DR}(\mathrm{HR}=1.96)$. Myometrial invasion was selected for LRR, DR, and DFS but was not associated with OS. Invasion of cornuae was not selected and was therefore left out of the nomogram. LVSI was selected for all outcomes $(\mathrm{HR}=1.1-1.2)$. All demographic and clinical predictors had HR of $>1$, meaning that higher age, higher grade, and myometrial invasion or increase in invasion depth are associated with a worse prognosis.

Treatment variables were associated with LRR and DFS (HR $<1$ : both treatments improved these outcomes) but not with DR and OS. This implies that pelvic EBRT and VBT reduce LRR but do not result in improved OS, which is highly driven by age and distant recurrence: 5-year OS was
88.3\% for DR-free patients versus $18.2 \%$ for patients with DR. For LRR and DFS, the RT variables were selected, which suggests that for those outcomes, the observational patient group is an important reference group when reporting the association of RT modalities with outcome. The resulting nomograms (Fig. 1) are able to estimate outcome probabilities for each specific outcome by assigning a score (Fig. 1, upper scale "Score") to each predictor value. The sum of these scores corresponds to an event probability.

\section{Nomogram validation}

Performances of the nomograms are reported in Table 4 for both the training set $(\mathrm{N}=1240)$ and the 2 validation sets $(\mathrm{n}=244$ and 291). Highest training c-indices were reported for LRR $(0.78$; $95 \%$ confidence interval $[\mathrm{CI}]$ : 0.73-0.83) and DR $(0.76 ; 95 \%$ CI: $0.71-0.81)$. The performance for DR was reproduced in the validation datasets, with c-indices of 0.64 and 0.76 , and 0.73 for the merged set. However, lower c-indices of $0.58(0.44-0.71)$ and 0.62 (0.49-0.75) were found for LRR. The survival-related nomograms validated with significant consistency; for DFS, the c-index was 0.71 (95\% CI: 0.67-0.74) for training and 0.69 (95\% CI: $0.63-0.75)$ for validation. For OS, the c-index was 0.71 (95\% CI: 0.67-0.75) for training, and this validated very well with c-index of 0.70 (95\% CI: 0.64 0.76).

Figure 2 shows that the nomograms have a good calibration when tested in both the training and the validation

Table 3 Median follow-up and outcome rates at 5 and 10 years for training and validation datasets

\begin{tabular}{|c|c|c|c|c|}
\hline & No. of events & $\begin{array}{l}\text { Median follow-up } \\
(\mathrm{mo})(95 \% \mathrm{CI})\end{array}$ & $\begin{array}{c}\text { Outcome } \% \text { at } 5 \mathrm{y} \\
(95 \% \mathrm{CI})\end{array}$ & $\begin{array}{c}\text { Outcome } \% \text { at } 10 \mathrm{y} \\
(95 \% \mathrm{CI})\end{array}$ \\
\hline \multicolumn{5}{|c|}{ Training ( $\mathrm{N}=1240$ patients) } \\
\hline VR & 59 & $72.4(66.5-79.4)$ & $4.43(3.16-5.68)$ & $5.63(4.05-7.19)$ \\
\hline PR & 34 & $74.9(69.8-82.4)$ & $2.38(1.42-3.33)$ & $2.95(1.8-4.09)$ \\
\hline LRR & 92 & $74.8(69.1-82.4)$ & $6.69(5.13-8.22)$ & $8.44(6.52-10.3)$ \\
\hline DR & 114 & $81.2(73.8-92.4)$ & $7.17(5.58-8.73)$ & $8.55(6.71-10.4)$ \\
\hline DFS & 428 & $120(113-126)$ & $79.8(77.3-82.3)$ & $67(63.8-70.3)$ \\
\hline OS & 393 & $139(135-143)$ & $83.7(81.4-86)$ & $70.9(67.8-74)$ \\
\hline \multicolumn{5}{|c|}{ Maastro $(n=244$ patients $)$} \\
\hline VR & 18 & $59.9(55-62.4)$ & $7.81(3.98-11.5)$ & $10.8(5.15-16.2)$ \\
\hline PR & 4 & $57.7(52.4-61.2)$ & $1.84(0.035-3.61)$ & $1.84(0.035-3.61)$ \\
\hline LRR & 21 & $59.9(55-62.3)$ & $9.16(5.06-13.1)$ & $12.2(6.33-17.7)$ \\
\hline DR & 17 & $59.9(55.4-62.4)$ & $7.15(3.39-10.8)$ & $11.2(4.39-17.4)$ \\
\hline DFS & 121 & $102(82.1-121)$ & $77.9(72.6-83.5)$ & $60.3(53.1-68.5)$ \\
\hline OS & 113 & $213(173-247)$ & $82.2(77.3-87.3)$ & $70(63.9-76.6)$ \\
\hline \multicolumn{5}{|c|}{ Enschede ( $n=291$ patients) } \\
\hline VR & 8 & $66.2(55.6-77.4)$ & $2.95(0.91-4.95)$ & $2.95(0.91-4.95)$ \\
\hline PR & 15 & $68.2(56-78.4)$ & $5.58(2.65-8.42)$ & $6.41(3.07-9.64)$ \\
\hline LRR & 19 & $69.1(56.9-80)$ & $6.94(3.75-10)$ & $7.76(4.2-11.2)$ \\
\hline DR & 40 & 73 (66.4-87) & $13.2(8.94-17.3)$ & $16(11-20.7)$ \\
\hline DFS & 90 & $92.3(77.9-116)$ & $75.9(70.7-81.5)$ & $63.9(57.4-71.2)$ \\
\hline OS & 81 & $90.4(77.9-116)$ & $79.1(74-84.5)$ & $65.7(59-73)$ \\
\hline
\end{tabular}

Abbreviations: $\mathrm{DFS}=$ disease-free survival; $\mathrm{DR}=$ distant relapse; $\mathrm{LRR}=$ locoregional relapse; $\mathrm{OS}=$ overall survival; $\mathrm{PR}=$ pelvic relapse; $\mathrm{VR}=$ vaginal relapse. 
Table $4 \quad \mathrm{HR}$ and corresponding $P$ values based on multivariate Cox models for selected prognostic factors with respect to the different endpoints*

\begin{tabular}{|c|c|c|c|c|c|c|c|c|}
\hline Factor & \multicolumn{2}{|c|}{ LRR } & \multicolumn{2}{|c|}{ DR } & \multicolumn{2}{|c|}{ DFS } & \multicolumn{2}{|c|}{ OS } \\
\hline Age & 1.35 & .006 & 1.35 & .001 & 1.77 & $<.001$ & 1.94 & $<.001$ \\
\hline Myometrial invasion & 1.50 & .001 & 1.26 & .033 & 1.11 & .045 & - & - \\
\hline Invasion of cornuae & - & - & - & - & - & - & - & - \\
\hline Vascular invasion & 1.21 & .029 & 1.21 & .007 & 1.13 & .007 & 1.12 & .016 \\
\hline VBT & 0.64 & $<.001$ & - & - & 0.82 & .009 & - & - \\
\hline Training c-index $(95 \% \mathrm{CI})$ & \multicolumn{2}{|c|}{$0.78(0.73-0.83)$} & \multicolumn{2}{|c|}{$0.76(0.71-0.81)$} & \multicolumn{2}{|c|}{$0.71(0.67-0.74)$} & \multicolumn{2}{|c|}{$0.71(0.67-0.75)$} \\
\hline Validation Maastro c-index $(95 \% \mathrm{CI})$ & \multicolumn{2}{|c|}{$0.58(0.44-0.71)$} & \multicolumn{2}{|c|}{$0.64(0.49-0.78)$} & \multicolumn{2}{|c|}{$0.69(0.61-0.77)$} & \multicolumn{2}{|c|}{$0.71(0.62-0.78)$} \\
\hline Validation Enschede c-index (95\% CI) & \multicolumn{2}{|c|}{$0.62(0.49-0.75)$} & \multicolumn{2}{|c|}{$0.76(0.68-0.84)$} & \multicolumn{2}{|c|}{$0.70(0.62-0.78)$} & \multicolumn{2}{|c|}{$0.70(0.63-0.77)$} \\
\hline Validation merged c-index (95\% CI) & \multicolumn{2}{|c|}{$0.59(0.50-0.68)$} & \multicolumn{2}{|c|}{$0.73(0.65-0.80)$} & \multicolumn{2}{|c|}{$0.69(0.63-0.75)$} & \multicolumn{2}{|c|}{$0.70(0.64-0.76)$} \\
\hline
\end{tabular}

datasets. Only predictions for LRR were significantly underestimated for the lower risk patients $(P<.0001)$. Application of the DFS and OS nomograms to the training and validation datasets, respectively, are shown in supplementary Figure E3 (available online at www. redjournal.com). The median risk probability was used as a threshold to distinguish between high- and low-risk patients. For both outcomes and both datasets, the KaplanMeier curves were significantly distinct. This confirms the fact that the nomograms validate well and that they have the potency to distinguish among risk groups based on a multivariate set of predictors.

\section{Use of the nomograms in clinical practice}

Examples of the potential use of the nomograms during patient counseling have been described in supplementary Table E5 (available online at www.redjournal.com). This shows that the nomograms are especially valid for use when counseling patients with intermediate- and highintermediate-risk stage I endometrial carcinoma (the risk groups on which the nomograms have been built). For patients with all high-risk factors (advanced age, grade 3 , LVSI, deep invasion), the model may slightly overestimate events.

\section{Discussion}

This pooled analysis of outcome data of 1240 EC patients treated in the randomized PORTEC-1 and PORTEC-2 trials was done to construct nomograms which could be used to predict long-term outcome after surgery with or without pelvic EBRT or VBT. Because of the random treatment allocation, the complete follow-up information, and the large number of patients, the pooled trial databases provided unique data with which to reliably analyze such outcomes. The nomograms are able to accurately predict long-term outcome in terms of LRR, DR, and survival with or without adjuvant treatment and have been externally validated for survival.

All of the previously developed nomograms were able to predict the likelihood of relapse and survival based on prognostic factors. For example, the Australian nomogram application is used in clinical practice for discussing individual patients' risks of disease recurrence based on clinical-pathological prognostic factors (12). Likewise, the Mayo Clinic nomogram is used to counsel a patient on the risks and benefits of second staging surgery based on the patient's individual risk of having microscopic pelvic lymph node metastases (13).

The nomograms provided in this study are unique in that they are the first set of nomograms that include information for outcomes with or without adjuvant EBRT or VBT. With this inclusion of adjuvant treatment, these nomograms are the ideal basis for individual patient information and counseling. Both can be used after endometrial tissue sampling and/or after definitive surgery for shared decision making in daily clinical practice.

Decisions about the minimal benefit required to justify adjuvant therapy as made by local or national guideline committees do not allow for such individual preferences and informed decisions. In view of the absence of a survival benefit, RT for early stage EC (with or without risk factors) was uniformly abandoned in Denmark (17). Recent results of a Danish cancer registry study show that without adjuvant pelvic EBRT or brachytherapy, 22\% of EC patients with intermediate risk factors will experience a relapse, and $14 \%$ of the $22 \%$ will have a locoregional recurrence (18). PORTEC-2 data suggest that the majority of these recurrences (12\% of these $14 \%$ ) could have been prevented with VBT (5). Patients should be aware of these facts and 
A

Score

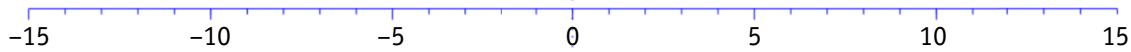

Age (years)

Radiotherapy

$\begin{array}{lllllllllllll}35 & 40 & 45 & 50 & 55 & 60 & 65 & 70 & 75 & 80 & 85 & 90 & 95\end{array}$

FIGO grade

$\begin{array}{llll}\text { EBRT } & \text { VBT } & \text { none }\end{array}$

Myometrial invasion depth

Vascular invasion

\begin{tabular}{lll}
\hline 11 & G2 & G3
\end{tabular}

$<50 \% \quad>50 \%$

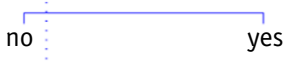

Sum of scores

\begin{tabular}{lllllll}
\hline-20 & -10 & 0 & 10 & 20 & 30 & 40
\end{tabular}

Locoregional relapse

within 5 years [\%] 1

$5 \quad 10$

20

$\begin{array}{lllllll}30 & 40 & 50 & 60 & 70 & 80 & 90\end{array}$

B

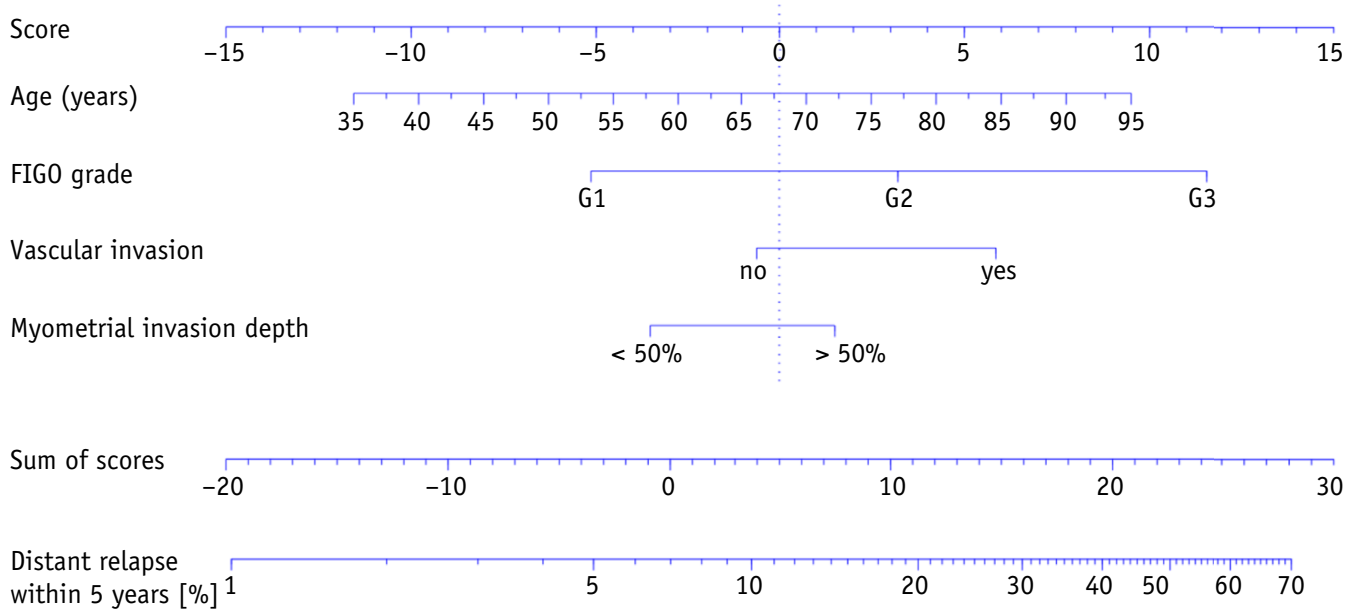

Fig. 1. Nomograms for (A) locoregional recurrence, (B) distant relapse, (C) disease-free survival, and (D) overall survival based on the final trial cohort (with revised pathology data). EBRT and VBT should not be added (as the series did not include patients who had both) but used separately. EBRT = external beam radiation therapy; FIGO = International Federation of Gynecology and Obstetrics; $\mathrm{G}=$ grade; VBT = vaginal brachytherapy.

of the pros and cons of such treatment decisions and should be able to make their own informed choices (9). In a specific treatment preference study, EC patients reported a relatively low minimally desired benefit of VBT $(0 \%-8 \%)$ at which they would prefer VBT over a watchful waiting approach (10). The current nomogram would be an easy and understandable instrument to be used in shared decision making, to facilitate understanding of the individual risk profile, and to discuss whether pelvic EBRT or VBT would be useful for the specific situation. In other cancer types, where the decision to use adjuvant treatment is based on risk factors, such as in breast cancer, prediction models such as the Web-based program Adjuvant! Online have been implemented in clinical practice worldwide (19). Using such support systems greatly facilitates patient understanding of risks and benefits of adjuvant therapy, and having such models for EC would facilitate shared decision making and also tailor the use of health care resources to the higher risk groups. In addition, the nomograms can also be used for selection of patients for clinical trials of adjuvant treatments and for generation of new hypotheses.

A limitation of our study is the relatively limited number of patients in the validation groups and the fact that the validation groups had less performance in the validation of LRR. Both the fact that the 2 validation sets did not include patients with (high) intermediate risk factors who were treated with surgery alone, and the relatively low number of LRR events contributed to the lower c-index. However, the performance of the validation for DR, DFS, and OS was excellent. We aimed to avoid overfitting by reducing the amount of predictors in the nomogram, by using the backward feature selection method. 


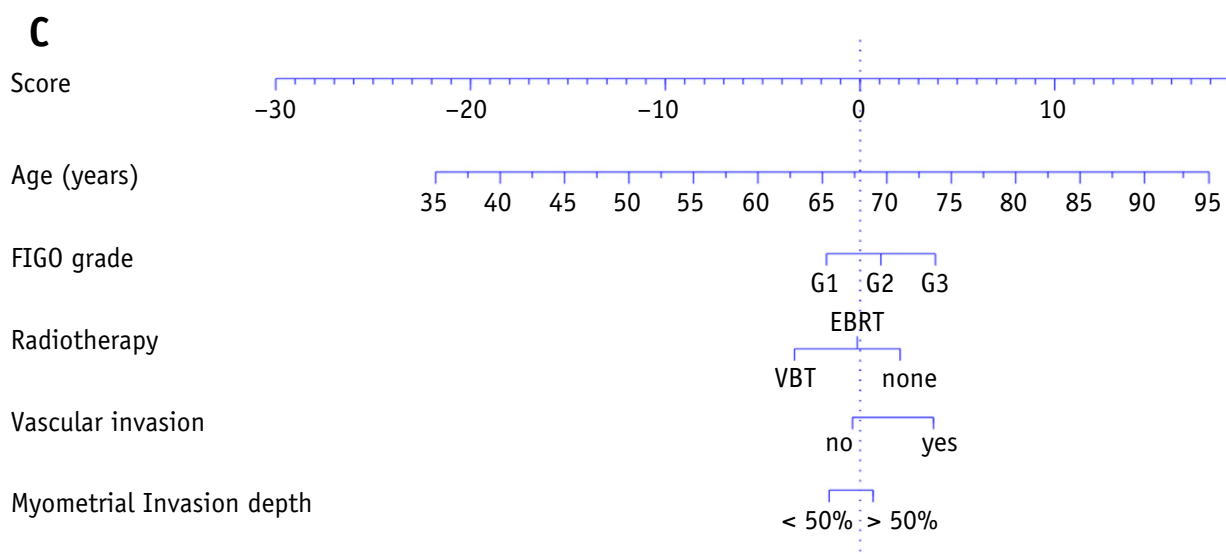

Sum of scores
$\begin{aligned} & \text { Relapse or death } \\ & \text { within } 5 \text { years [\%] }\end{aligned}$

D

\begin{tabular}{|c|c|c|c|c|c|c|c|c|c|c|c|c|c|c|c|}
\hline Score & -30 & & -2 & & & $\begin{array}{c}1 \\
-10\end{array}$ & & & 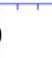 & & $\begin{array}{l}1 \\
10\end{array}$ & & & 20 & 30 \\
\hline Age (years) & & 35 & 40 & 45 & 50 & 55 & 60 & 65 & 70 & 75 & 80 & 85 & 90 & 95 & \\
\hline FIG0 grade & & & & & & & & G1 & G2 & $\vec{G} 3$ & & & & & \\
\hline Vascular inv & & & & & & & & $\mathrm{n}$ & & yes & & & & & \\
\hline
\end{tabular}

Sum of scores
$\begin{aligned} & \text { Death } \\ & \text { within } 5 \text { years }[\%]\end{aligned} \quad-20$
10

Fig. 1. Continued.

Another limitation is that the nomograms should be interpreted with caution for patients who have higher risk factors other than the large pooled group of patients with (high-) intermediate-risk stage I endometrial carcinoma on which the nomograms have been built, as has been illustrated in supplementary Table E5 (available online at www. redjournal.com). For patients who have all high-risk factors (advanced age, grade 3, LVSI, deep invasion), the model seems to somewhat overestimate recurrence rates.

The fact that many EC patients have comorbidities and approximately $50 \%$ of deaths are due to causes other than EC is well known. Still, it is relevant when discussing adjuvant treatment with a patient to know how the likelihood of survival with and without relapse will be; in future, such information could be added using the patient's comorbidity profile as also used in other prediction models such as Adjuvant! (19). Combined analysis and validation with the Australian nomogram (13) is being planned.
A limitation of all current nomograms are that they take into account only classic clinicopathological risk factors. They only give a rough estimate of the patient's expected outcome. In our analysis, the classic risk factors increasing age, higher FIGO grade, and presence of LVSI were consistently selected for all outcomes, LRR, DR, DFS, and OS. For LRR, which is the main outcome that is influenced by pelvic EBRT and VBT in early stage EC, we found FIGO grade to have the highest HR, and grade was of stronger significance than LVSI and depth of myometrial invasion. Invasion of the cornuae, where the myometrium is thinnest and myometrial invasion is usually relatively close to the serosa but which may not be consistently reported in pathology reports, had the lowest impact and could be omitted. Both this and recent analyses suggest that LVSI may be the strongest factor for DR and survival (especially if extensive LVSI is taken into account), and the prognostic information of LVSI might be at least as strong as that of microscopic nodal involvement (20-22). 

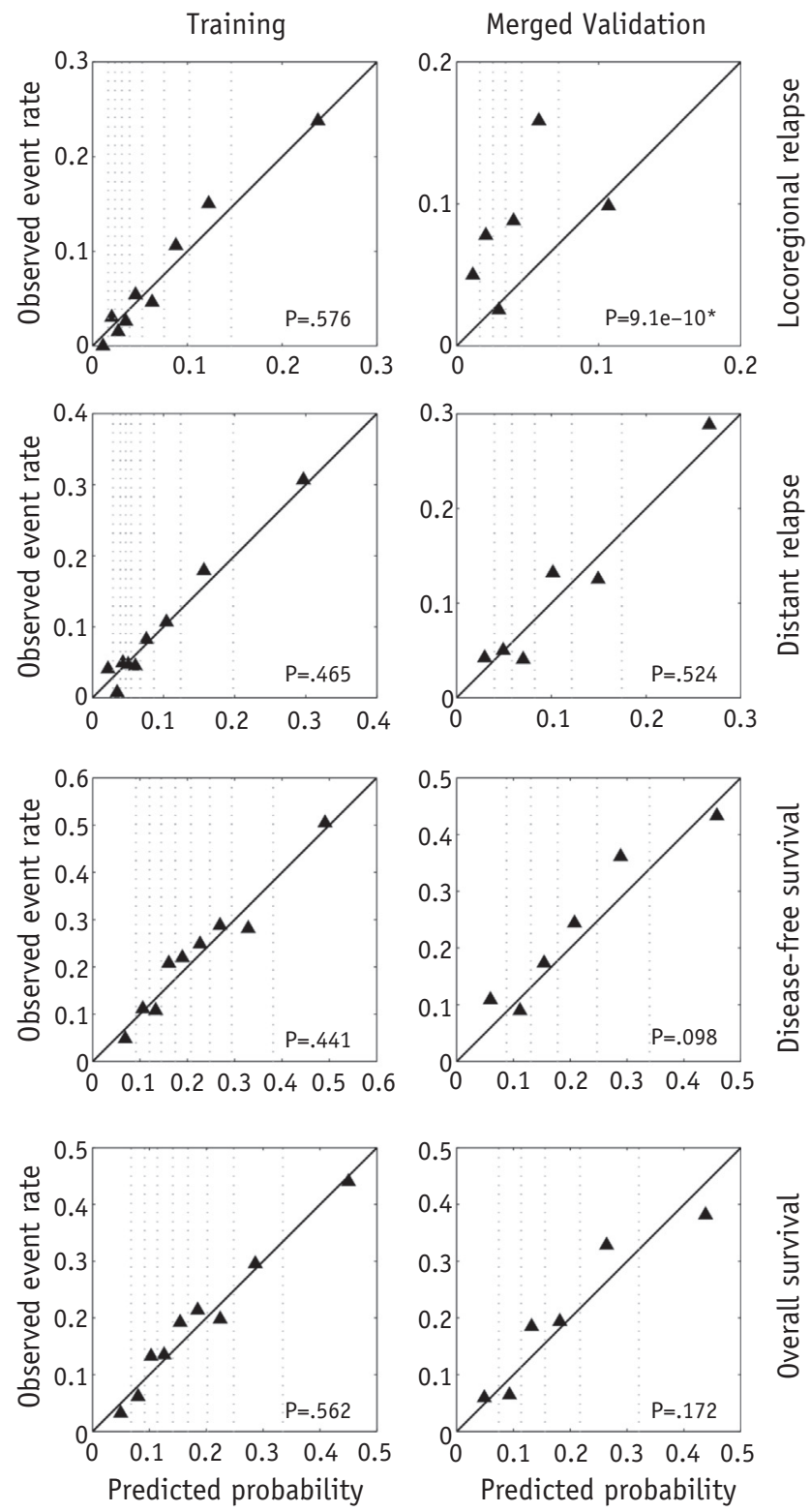

Fig. 2. Calibration plots show predicted event rates versus observed event rates for equally sized subgroups of patients. There are 9 subgroups for the training datasets and 6 for the merged validation dataset. Hosmer-Lemeshow statistic was used to compute $P$ values for significant under- or overestimation of observed probabilities by the nomograms.

\section{Conclusions}

Although these nomograms are very useful in current risk prediction and for shared decision making about adjuvant treatment, addition of molecular genetic risk factors such as L1 cell adhesion molecule (L1CAM) expression, POLE proofreading mutations, TP53 mutation, microsatelliteinstability (MSI) and combinations thereof $(7,8)$ or of the rhw molecular signature (23) in risk prediction models might lead to much greater precision of the risk estimates, and in future nomograms, such features should be incorporated. These nomograms for use in patient counseling and shared decision making are able to accurately predict long-term outcome in terms of locoregional recurrence, distant recurrence, and survival with or without adjuvant EBRT or VBT.

\section{References}

1. Blake P, Swart AM, Orton J, et al. Adjuvant external beam RT in the treatment of endometrial cancer (MRC ASTEC and NCIC CTG EN.5 randomised trials): Pooled trial results, systematic review, and metaanalysis. Lancet 2009;373:137-146.

2. Kong A, Johnson N, Kitchener HC, et al. Adjuvant radiotherapy for stage I endometrial cancer: An updated Cochrane systematic review and meta-analysis. J Natl Cancer Inst 2012;104;21:1625-1634.

3. Creutzberg CL, van Putten WLJ, Koper PC, et al. Survival after relapse in patients with endometrial cancer: results from a randomized trial. Gynecol Oncol 2003;89:201-209.

4. Onsrud M, Cvancarova M, Hellebust TP, et al. Long-term outcomes after pelvic radiation for early-stage endometrial cancer. J Clin Oncol 2013:31:3951-3956.

5. Nout RA, Smit VT, Putter H, et al. Vaginal brachytherapy versus pelvic external beam radiotherapy for patients with endometrial carcinoma of high-intermediate risk (PORTEC-2): an open-label, noninferiority, randomised trial. Lancet 2010;375:816-823.

6. Nout RA, Putter H, Jurgenliemk-Schulz IM, et al. Five-year quality of life of endometrial cancer patients treated in the randomised Post Operative Radiation Therapy in Endometrial Cancer (PORTEC-2) trial and comparison with norm data. Eur J Cancer 2012;48:1638-1648.

7. Nout RA, Bosse T, Creutzberg CL, et al. Improved risk assessment of endometrial cancer by combined analysis of MSI, PI3K-AKT, Wnt/sscatenin and P53 pathway activation. Gynecol Oncol 2012;126:66-73.

8. Zeimet AG, Reimer D, Huszar M, et al. L1CAM in early-stage type I endometrial cancer: Results of a large multicenter evaluation. J Natl Cancer Inst 2013;105:1142-1150.

9. Pieterse AH, Baas-Thijssen MC, Marijnen CA, et al. Clinician and cancer patient views on patient participation in treatment decisionmaking: A quantitative and qualitative exploration. $\mathrm{Br} J$ Cancer 2008;99:875-882.

10. Kunneman M, Pieterse AH, Stiggelbout AM, et al. Treatment preferences and involvement in treatment decision making of patients with endometrial cancer and clinicians. Br J Cancer 2014;111:673-679.

11. Abu-Rustum NR, Zhou Q, Gomez JD, et al. A nomogram for predicting overall survival of women with endometrial cancer following primary therapy: Toward improving individualized cancer care. Gynecol Oncol 2010;116:399-403.

12. Kondalsamy-Chennakesavan S, Yu C, Kattan MW, et al. Nomograms to predict isolated loco-regional or distant recurrence among women with uterine cancer. Gynecol Oncol 2012;125:520-525.

13. Alhilli MM, Podratz KC, Dowdy SC, et al. Risk-scoring system for the individualized prediction of lymphatic dissemination in patients with endometrioid endometrial cancer. Gynecol Oncol 2013;131:103-108.

14. Creutzberg CL, van Putten WL, Koper PC, et al. Surgery and postoperative radiotherapy versus surgery alone for patients with stage-1 endometrial carcinoma: multicentre randomised trial. PORTEC Study Group. Post Operative Radiation Therapy in Endometrial Carcinoma. Lancet 2000;355:1404-1411.

15. Creutzberg CL, Nout RA, Lybeert ML, et al. Fifteen-year radiotherapy outcomes of the randomized PORTEC-1 trial for endometrial carcinoma. Int J Radiat Oncol Biol Phys 2011;81:e631-e638.

16. Creutzberg CL, van Putten WL, Warlam-Rodenhuis CC, et al. Outcome of high-risk stage IC, grade 3, compared with stage I endometrial carcinoma patients: the Postoperative Radiation Therapy in Endometrial Carcinoma Trial. J Clin Oncol 2004;22:1234-1241.

17. Bertelsen K, Ortoft G, Hansen ES. Survival of Danish patients with endometrial cancer in the intermediate-risk group not given 
postoperative radiotherapy: The Danish Endometrial Cancer Study (DEMCA). Int J Gynecol Cancer 2011;21:1191-1199.

18. Ortoft G, Hansen ES, Bertelsen K. Omitting adjuvant radiotherapy in endometrial cancer increases the rate of locoregional recurrences but has no effect on long-term survival: The Danish Endometrial Cancer Study. Int J Gynecol Cancer 2013;23:1429-1437.

19. Ozanne EM, Braithwaite D, Sepucha K, et al. Sensitivity to input variability of the Adjuvant! online breast cancer prognostic model. $J$ Clin Oncol 2009;27:214-219.

20. Narayan K, Khaw P, Bernshaw D, et al. Prognostic significance of lymphovascular space invasion and nodal involvement in intermediate- and high-risk endometrial cancer patients treated with curative intent using surgery and adjuvant radiotherapy. Int J Gynecol Cancer 2012;22:260-266

21. Briet JM, Hollema H, Reesink N, et al. Lymphvascular space involvement: An independent prognostic factor in endometrial cancer. Gynecol Oncol 2005;96:799-804.

22. Cohn DE, Horowitz NS, Mutch DG, et al. Should the presence of lymphvascular space involvement be used to assign patients to adjuvant therapy following hysterectomy for unstaged endometrial cancer? Gynecol Oncol 2002;87:243-246.

23. Kandoth C, Schultz N, Cherniack AD, et al. Integrated genomic characterization of endometrial carcinoma. Nature 2013;497: $67-73$. 